Théologiques

Théologiques

\title{
Le discernement dans les Exercices spirituels d'Ignace de Loyola
}

\section{Guido Mongini}

Volume 22, numéro 2, 2014

Le discernement spirituel

URI : https://id.erudit.org/iderudit/1035688ar

DOI : https://doi.org/10.7202/1035688ar

Aller au sommaire du numéro

\section{Éditeur(s)}

Faculté de théologie et de sciences des religions, Université de Montréal

\section{ISSN}

1188-7109 (imprimé)

1492-1413 (numérique)

Découvrir la revue

Citer cet article

Mongini, G. (2014). Le discernement dans les Exercices spirituels d'Ignace de Loyola. Théologiques, 22(2), 99-135. https://doi.org/10.7202/1035688ar
Résumé de l'article

L'article traite de la question du discernement intérieur dans la pensée du fondateur de la Compagnie de Jésus, Ignace de Loyola. Malgré l'existence d'une vaste littérature sur le discernement ignacien, certains problèmes demeurent, notamment celui de la relation entre la théorie du discernement, exposée dans les Exercices spirituels d'Ignace, et la pratique. L'article a pour but de retracer le système des " règles du discernement » dans les Exercices, en clarifiant certains malentendus herméneutiques concernant les concepts (surtout en référence à la lecture faite par Roland Barthes) et l'histoire du XVI $\mathrm{e}^{\mathrm{e}}$ siècle (où l'expérience religieuse d'Ignace de Loyola trouve ses racines). Aux alentours de 1550, la méthode du discernement proposée dans les Exercices a suscité de nombreuses polémiques, notamment en Espagne. Ici, à la question de l'« élection ", qui requiert la mise en pratique du discernement, s'est ajoutée celle de la relation entre spiritualisme, hérésie et orthodoxie, jusqu'à la remise en question des fondements de l'expérience religieuse jésuite dans son ensemble. 


\title{
Le discernement dans les Exercices spirituels d'Ignace de Loyola
}

\author{
Guido Mongini* \\ Università di Padova (Italie)
}

\section{Ruptures et continuités}

Des multiples paradoxes qui caractérisent l'histoire des origines de la Compagnie de Jésus et de son fondateur Ignace de Loyola, encore faut-il y ajouter le discernement spirituel. D'une part, les techniques et les méthodes enseignées par Loyola dans ses célèbres Exercices spirituels ont eu - d'abord par l'entremise des Exercices eux-mêmes, et dans un second temps, à travers une multitude de canaux, à l'intérieur et à l'extérieur de la Compagnie une importance et une diffusion exceptionnelles dans le monde catholique tout au long de l'histoire moderne et contemporaine; et s'étendant, en plus, dans le sillage des missions étrangères des jésuites, dans la grande majorité des pays du globe. Ainsi, l'importance culturelle des théories du discernement décrites par Loyola ne peut être sous-estimée, et ce, tant sur le plan chronologique que sur le plan géographique. Durant des siècles, elles ont gravé et modelé en profondeur l'histoire même du discernement intérieur. Soit qu'elles aient été admises au-delà de la Compagnie de Jésus et interprétées différemment ou réadaptées dans les divers ordres religieux catholiques, donnant naissance à une vaste littérature pieuse et «spirituelle» entre le XVII ${ }^{\mathrm{e}}$ et le XVIII ${ }^{\mathrm{e}}$ siècle; soit qu'elles aient plutôt été neutralisées ou regardées avec

* Guido Mongini conduit ses recherches auprès de l'Université de Padoue, sur plusieurs axes: a) histoire des origines de la Compagnie de Jésus et histoire de l'identité jésuite (XVI-XIX siècles); b) hérésies et hérétiques au XVI ${ }^{\mathrm{e}}$ siècle; c) histoire de la mystique (XVI ${ }^{\mathrm{e}}-\mathrm{XX}^{\mathrm{e}}$ siècles). Il travaille actuellement sur un riche dossier de lettres indipetae, avec lesquelles les jésuites sollicitaient leur envoi dans les missions étrangères. Il a récemment publié une biographie grand public sur Ignace de Loyola: (2014) Ignazio di Loyola. Un illuminato al servizio della Chiesa, Milan, Edizioni del Sole24Ore. 
suspicion comme étant un des moyens principaux avec lesquels les jésuites contrôlaient les consciences tant des fidèles que des régnants, des rois et des élites ${ }^{1}$ de la société d'Ancien Régime ${ }^{2}$.

D'un autre côté, bien qu'une abondante littérature, encore récemment $^{3}$, ait mis en évidence les similitudes et les analogies avec d'autres exemples appartenant surtout à la tradition du monachisme, il importe de souligner que les règles de la discretio spirituum insérées par Loyola dans les Exercices spirituels ne dépendent pas de l'une ou de l'autre tradition, mais sont le produit exclusif de l'expérience religieuse personnelle du fondateur de la Compagnie de Jésus. Quant au discernement intérieur, de tout ce qui a été enseigné par les moines de l'Antiquité, tant latins que byzantins, et transmis par les maîtres médiévaux — sans pour autant rien retirer aux possibles et incontestables analogies - il est toutefois indispensable de réaffirmer l'indépendance des théories de Loyola de toutes traditions, afin de ne pas perdre l'authentique dimension historique tant de son expérience religieuse que, conséquemment, des règles du discernement qu'il énonce. Il convient de soustraire ces règles à une illusoire continuité l' " histoire de la spiritualité » — qui risque continuellement, mettant en sourdine les ruptures et les discontinuités de l'histoire, et de ses contextes, de retrouver partout les mêmes objets et les mêmes modalités, dans un rassurant retour de l'identique qui semble être suffisant pour confirmer une tradition. Au contraire, nées d'une particulière et très controversée expérience religieuse - celle d'un ex-soldat basque, Ignace de Loyola, dans l'Espagne de la première moitié du XVI ${ }^{\mathrm{e}}$ siècle, parcourue par des effervescences souvent subversives des formes traditionnelles de la dévotion et de la vie spirituelle, de l'érasmisme au luthéranisme, aux alumbrados castillans ${ }^{4}$ - les règles du discernement ignacien constituèrent, historiquement, une rupture par rapport à la tradition, non pas tant sur le plan des contenus que sur celui de leurs origines et, donc, de leur statut, et instaurèrent, à leur tour, une tradition, tant par l'importance qu'elles eurent à l'intérieur de la Compagnie de Jésus que par leur diffusion hors de celle-ci, comme il a déjà été mentionné. De même, on le verra ensuite, les potentialités, pour

1. En français dans le texte.

2. En français dans le texte.

3. Dans Plazaola (1998), de nombreuses contributions s'occupent des règles du discernement et des possibles sources.

4. Sur les fondements historiques de l'expérience religieuse d'Ignace de Loyola et sur les suspicions et conflits auxquels elle donna lieu, voir Mongini (2011), où se trouvent des renvois aux sources bibliographiques les plus importantes. 
ainsi dire, des règles du discernement de Loyola furent considérablement développées du fait de leur disposition dans les Exercices spirituels: non pas «corps» isolé et détaché du reste, mais noyau fondamental de tout le parcours des Exercices. Les règles ignaciennes en suivirent la diffusion et la fortune, atteignant de larges et diversifiées strates de la société - également populaire ${ }^{5}$ - et soulevant, entre-temps, et justement dans les débuts de la Compagnie, les suspicions de l'Inquisition. Ainsi, continuité et discontinuité, rupture dans la tradition et nouvelles instaurations se superposent dans l'histoire des théories du discernement d'Ignace de Loyola, et sont à la base de leur succès depuis plusieurs siècles tout comme les relectures continues et les réactualisations pastorales, de nos jours, encore existantes dans l'hétérogène monde catholique ${ }^{6}$.

\section{Théorie du discernement ignacien: les Exercices spirituels et les règles du discernement}

La rédaction, en espagnol, du texte des Exercices spirituels, fut longue et complexe, et ses prémisses remontent - comme Ignace l'affirme dans son Autobiographie - à la période de sa conversion, alors que, convalescent des suites d'une blessure rapportée de la défense de Pampelune, il réside dans la maison familiale à Loyola et commence l'examen de ses pensées et de ses états d'âme. Sans pouvoir s'arrêter sur l'histoire des diverses phases rédactionnelles des Exercices ${ }^{7}$ et sur leurs circonstances, il est nécessaire, en revanche, de fournir quelques dates essentielles sur leur structure, à partir du texte officiel, approuvé par le Pape Paul III en 1548.

5. Un exemple significatif (un parmi tant d'autres) de tentative de diffusion auprès de vastes cercles de lecteurs laïcs des techniques du discernement élaborées par Loyola a été effectué par de Ribadeneira (1589, 271-77); voir à ce sujet Mongini (2012, 189-195).

6. Les Exercices spirituels et les règles du discernement qui figurent à l'intérieur ont donné origine à une immense littérature imprimée à des fins pastorales et d'accompagnement spirituel; on se limite à indiquer à titre d'exemple, bien que n'étant pas sans intérêts historiques plus généraux, le texte de Jurado (1997). On peut également consulter, dans une perspective d'actualisation du discernement ignacien, de Vries (1979).

7. Les principales rédactions (une en espagnol et trois latines) des Exercices sont dans Loyola (1969) (duquel on cite d'ordinaire, à partir de la version espagnole dite "autographe ", indiquant non pas les pages mais les paragraphes du texte). De nouvelles études, en cours, apportent des indications précieuses (bien que n'étant pas conclusives) sur la question cruciale de la datation du texte définitif, voir Loyola (2000). 
Dans cette première édition imprimée ${ }^{8}$, en latin, les Exercices comptent 230 pages et sont subdivisés en quelques grandes sections: les Annotations introductives, quatre cycles d'exercices (les légendaires Semaines), les indications sur les "trois manières de prier ", une sélection de passages/extraits bibliques (les «Mystères de la vie de notre Seigneur»), une section sur les règles pour un discernement des esprits (sur laquelle on reviendra par la suite), suivie de prescriptions sur les aumônes, sur les scrupules et "pour sentir vraiment avec l'Église orthodoxe». Les célèbres "compositions visuelles de lieu' " sont particulières aux divers "exercices » des quatre Semaines. Elles consistent en exercices d'imagination mentale - aussi définis comme "méditations" — sur un thème précis et fourni à l' "exercitant» par le directeur des exercices. Le thème peut être un petit verset évangélique qui narre un épisode de la vie du Christ, ou alors, comme dans les deux premières Semaines, une «situation» particulière, par exemple l'enfer (Loyola 1969, $\mathbb{S} 65-71$ ), avec les damnés immergés dans le feu, ou d'autres sujets en lien avec le parcours de conversion de l'exercitant, comme la célèbre méditation des « deux étendards " (Loyola 1969, \$136147) dans laquelle l'exercitant doit imaginer un grand champ où sont rangées deux armées, la première commandée par le Christ et l'autre par Lucifer, et choisir de quel côté se trouver. À propos de la méditation qui prend appui sur la composition visuelle des lieux, Loyola a également prévu d'autres actions de la part de l'exercitant, lequel doit s'immerger, et d'une certaine manière "entrer ", dans la scène qu'il a imaginée intérieurement et y participer activement, c'est-à-dire sentir qu'il en fait partie, jusqu'à la "vivre», faisant participer, outre les forces internes (intellect, mémoire et volonté), également les cinq sens, s'efforçant d' "écouter ce que disent» (Loyola 1969, $\mathbb{1 2 3}$ ) les personnages de la scène imaginée, ou ressentir la chaleur du feu infernal, ou encore percevoir avec le toucher, l'odeur et la vue les détails corporels, matériels, de la composition visuelle. De cette façon, Loyola s'efforçait de réaliser une véritable convocation de toutes les composantes de l'être humain, y compris le corps (à propos duquel Ignace a fourni beaucoup d'indications, de la nourriture à l'habillement, aux postures, etc.) : l'exercitant parvenait à «habiter» et à vivre dans la scène imaginée. En ce sens, les diverses méditations des Exercices s'occupaient d'engager l'entièreté de l'individu dans le chemin de l'expé-

8. Reproduite dans l'appendice 2 de Plazaola (1998).

9. L'étude la plus approfondie sur la composition visuelle de lieu est de Fabre (1992). 
rience religieuse, et non pas seulement son imagination, comme il est possible, à tort, de supposer ${ }^{10}$.

Bien que les Exercices présentent une structure très articulée, leur fonctionnement est en revanche encore plus complexe. Afin de pouvoir comprendre, même partiellement, leur nature spécifique, il est nécessaire d'introduire une distinction de fond entre le texte comme ensemble d'indications et de prescriptions, le texte statique (dont on a d'ailleurs décrit synthétiquement la structure), et le texte dynamique ou agité, à savoir le texte qui doit être concrètement mis en acte, vécu et expérimenté, par celui qui fait les exercices, l'exercitant, sous la guidance de celui qui donne les exercices, le directeur des exercices ${ }^{11}$. Ce point capital met en lumière la particularité des Exercices ignaciens: en tant qu'objet-livre, ils ne sont pas destinés à la lecture, mais à la pratique. De plus, le texte véritable, le livre, n'est pas physiquement manipulé ni connu dans son intégralité par l'exercitant, mais seulement par le directeur qui lui en explique et prescrit les différents exercices. De la sorte, celui-ci n'a pas une vision complète du parcours des Exercices, que seul connaît le directeur. Par conséquent, émerge clairement la différence entre un lecteur hypothétique qui lit les Exercices comme un livre quelconque, et la fonction du même texte dans le cadre de sa spécifique destination religieuse. Dans ce cas, le lecteur se dédouble et se polarise, d'une certaine manière, entre le directeur des exercices (qui possède le livre, le connaît intégralement et l'explique petit à petit à l'exercitant) et l'exercitant même (qui connaît du texte seulement les passages que le directeur lui fournit oralement et qu'il doit mettre en pratique). Sans pouvoir s'arrêter ici sur les dispositions données par Loyola en ce qui concerne l'attitude que le directeur des Exercices devait avoir face à l'exercitant, il convient d'au moins souligner un point: à savoir qu'Ignace

10. Les quatre Semaines contiennent, outre de véritables exercices, d'autres éléments. Dans le détail, la première Semaine comprend le noyau théorique et théologique essentiel des Exercices, le "Principe et fondement »; les méthodes pour faire les examens de conscience particulier et général, et la confession. La seconde Semaine expose les indications sur les "états de vie » et les "modes d'humilité », mais, surtout, les méthodes sur les "façons de faire un choix", à savoir la fameuse élection, sur laquelle on reviendra. Une série de règles sur l'usage de la nourriture complète la troisième Semaine, alors que la quatrième comprend les "trois manières de prier » tout de suite après la «contemplation pour susciter en nous l'amour spirituel ». L'ensemble des quatre Semaines compose le mois intégral des Exercices.

11. Une distinction analogue, principalement articulée sur le plan structuraliste-linguistique et avec des orientations différentes, des différents niveaux du texte des Exercices a été effectuée par Barthes (1977, 25-64). 
avait beaucoup insisté sur la neutralité du rôle que devait tenir le directeur. Et ce, non seulement afin de ne pas influencer les choix et les décisions de l'exercitant, mais davantage pour consentir que la «communication» ou l' "échange» (comme s'exprimait Loyola) entre Dieu et l'exercitant se déroule de la façon la moins préméditée et la plus directe possible (c'était justement en référence au directeur qu'Ignace utilisait la célèbre métaphore de la "balance », le directeur devant précisément demeurer, comme celle-ci, "en équilibre au milieu ${ }^{12}$ ", Loyola $1969, \mathbb{S} 15$ ). Enfin, ce qui a été dit jusqu'à présent permet de définir les Exercices non pas comme un livre destiné à la lecture, mais bien à la pratique: comme une sorte de manuel, de recueil qui fournit à la fois des indications théoriques et opératives en relation à différentes modalités, phases et objectifs de l'expérience religieuse. Pour résumer, on peut affirmer que les Exercices de Loyola contenaient et en même temps pouvaient donner lieu à une grande variété d'expériences religieuses, à l'intérieur d'un horizon qui pouvait aller de la conversion d'une vie vécue dans le péché ou dans l'oubli de Dieu à un approfondissement radical, dans un sens spiritualiste, du chemin religieux, et à ce qu'Ignace définissait comme "communication entre les deux parties » (Loyola 1969, $\mathbb{2 3 1}$ ), à savoir le dialogue ou le contact direct, sans médiation, entre l'homme et la divinité, par voie d'inspiration intérieure ou d'illumination.

Laissant de côté leur signification générale, il faut maintenant s'arrêter sur un des éléments les plus caractéristiques des Exercices spirituels - et auquel se joint, dans la perspective ignacienne, le discernement — c'est-àdire l'attention pour la dimension psychologique de l'expérience religieuse. On ne peut faire qu'allusion, ici, d'un côté, à l'importance attribuée au corps par Ignace de Loyola, et de l'autre côté, à la centralité, dans les Exercices, de l'élément anthropologique constitué de la pensée (comprise dans toute son étendue de "mouvement intérieur» de la réflexion et du sentiment) comme élément à soumettre à l'analyse et à l'examen continu. En effet, tant l'action de penser que les pensées (pensamientos, moçiones) sont un sujet qui revient continuellement dans le texte ignacien ${ }^{13}$, au point où il est possible d'affirmer que ce sont précisément les pensées, et plus généralement la dimension mentale-émotionnelle-psychologique, qui

12. Avec les prémisses du paragraphe 14 .

13. Il n'y a pas moins, dans les Exercices, de 25 occurrences du terme "pensamiento ", alors que "pensar» revient 20 fois et la notion de "moçión" (motion intérieure) que Loyola considérait voisine à celle de "pensiamento » — revient 9 fois; voir Concordancia ignaciana (1996), sub voce. 
constituent le véritable et authentique matériel sur lequel converge toute l'activité intérieure de l'exercitant. De la sorte, le «travail sur les pensées » - soit en tant qu'actes de l'intellect et du raisonnement, soit en tant qu'effets ou contrecoups «internes », psychologiques, de la part des différents affects, passions, émotions, sentiments, perceptions, etc. qui surgissent de la conscience - la discipline de l'esprit et de l'âme, constituent un point cardinal dans le texte de Loyola ${ }^{14}$.

Précédemment, il a été mention de la composition visuelle de lieu et des choses «vues» intérieurement durant cette activité imaginative, l'exercitant - selon les prescriptions de Loyola - devait ensuite appliquer la réflexion et la volonté, mais également les affects, les émotions et les sentiments, et ses cinq sens de l'imagination. Donc, chacun des exercices représentait une convocation de l'entière psychologie de l'exercitant qui devait, de la sorte, se révéler complètement transformée - en effet, les Exercices avaient pour objectif de se "vaincre soi-même" (Loyola 1969, \$21) - selon un programme en deux étapes: "préparer et disposer l'âme à supprimer d'elle tous les affects/attachements mal ordonnés » et, successivement, "chercher et trouver la volonté divine dans l'organisation de sa vie» (Loyola 1969, $\mathbb{S} 1$ ). Sans parcourir dans les détails les nombreuses implications de la psychologie des Exercices, il est, en revanche, indispensable de s'arrêter sur les célèbres "Règles pour sentir et reconnaître de quelque façon les différents mouvements qui se produisent dans l'âme ", qui en constituent l'aspect le plus significatif. Par la suite, on examinera le rôle du discernement et son rapport à un des moments centraux des Exercices: le moment de l'élection, c'est-à-dire du choix.

Il est connu que le discernement spirituel, ou discretio spirituum, est un des éléments essentiels de la direction spirituelle et plonge ses racines dans une longue tradition qui dérive des origines mêmes du christianisme (voir, par exemple: 1Co 12,10; 1Jn 4,1) pour se développer ensuite dans le monachisme antique et médiéval. En regard de cette importante tradition, les deux séries de Règles sur le discernement d'Ignace de Loyola ne présentent pas, comme il a été mentionné, de grandes innovations. Leur particularité consiste, d'un côté, dans la lucidité et la clarté synthétique de

14. Sur un plan général, on peut observer que cette discipline de la pensée constitue le nécessaire correctif de la prodigieuse activité mentale mise en acte par l'exercitant dans les méditations, dans lesquelles les «compositions visuelles de lieu» ne sont pas autre chose que des exercices d'imagination accomplis avec l'esprit. En tant qu'actes prolongés de "pensée iconique ", c'est-à-dire orientés à produire des images, ils requièrent une capacité lucide de contrôle mental et volitif de la part de l'exercitant. 
l'exposition et leur insertion dans le cadre des Exercices, à savoir directement dans la pratique de l'exercitant, et de l'autre côté, par leurs connexions avec l'expérience religieuse illuministe et spiritualiste de Loyola.

Pour comprendre l'importance historique des règles sur le discernement, il importe de rappeler un fait essentiel, à savoir que l'expérience concrète de l'alternance des "esprits" effectuée par Loyola, au temps de sa conversion, constitue le noyau ancien duquel prit lentement forme la composition des Exercices ${ }^{15}$. En effet, il faut souligner que, selon le témoignage d'Ignace lui-même, la connaissance des procédés de la discretio spirituum ne lui provenait pas de la tradition précédente (à laquelle il ne faisait aucune référence), mais exclusivement d'une expérience personnelle d'illumination intérieure au cours de laquelle "comenzó a ver con otros ojos todas las cosas, $y$ a discernir y probar los espíritus buenos y malos ${ }^{16}$ " (Lainez 1943, 80). Durant cette période — à Manresa, août-septembre 1522 —, "Dieu se comportait avec lui comme le fait un maître d'école avec un enfant: il lui enseignait »; Ignace ajoutait ensuite qu' «il n'y en avait pas d'autre qui l'instruisait ${ }^{17}$ » (Loyola 1943, $\mathbb{S} 27$ ), cela pour souligner comment la matrice de ses expériences d'alors consistait exclusivement en son expérience spiritualiste personnelle.

Ainsi, produit exclusif de son expérience religieuse personnelle de nature illuministe, les règles du discernement étaient étroitement connectées à la structure des Exercices spirituels. En effet, si elles sont posées vers la fin du livre, elles renvoient en réalité à son début en ce qu'elles sont subdivisées en deux séries distinctes qui se réfèrent respectivement à la première et la seconde Semaines. L'importance que leur attribue l'auteur se manifeste par l'espace qu'elles occupent dans le texte, c'est-à-dire vingttrois paragraphes (Loyola 1969, $\mathbb{S} 313-336$ ): de la sorte, les quatorze premières règles sont "plus adaptées " (Loyola 1969, \$313) à la première Semaine, les huit règles successives concernent «surtout» (Loyola 1969, $\mathbb{S}$ 328) la seconde Semaine. Aussi, pour comprendre l'exact cadre de réfé-

15. Voir Acta Patris Ignatii (également nommé Le récit du pèlerin, la célèbre autobiographie de Loyola) dans Loyola (1943, 353-507).

16. "[Ignace] commença à voir, avec d'autres yeux, toutes les choses et à discerner et à mettre à l'épreuve les esprits bons et mauvais ». Ce témoignage de Lainez est parallèle à celui de Loyola (Loyola 1943, 404) où il se réfère à la célèbre «illumination du Cardoner" sans le détail sur le discernement. Sur l'importance et la signification de l'illumination du Cardoner pour Loyola, voir Mongini (2011, 63-65 ; 134-136).

17. De même pour la citation précédente. 
rence des deux séries de règles, faut-il rappeler comment la première Semaine est destinée à la conversion du pécheur, à savoir au passage, par l'entremise du repentir et de la pénitence, d'une vie mondaine au sens large (de la simple négligence ou de l'oubli de Dieu à la concrète pratique des péchés mortels) à une vie de réconciliation avec Dieu. Typiques de la première Semaine des Exercices, les méditations sur les péchés et sur l'enfer, dans lesquels - entre autres - l'exercitant expérimente les effets du châtiment éternel «vivant» la condition des damnés dans le feu infernal. Autrement dit, le but de la première Semaine est de faire réfléchir l'exercitant sur sa propre condition de pécheur et ses habitudes au péché, et sur son inattention vis-à-vis de la grâce divine. De la sorte, la première série de règles du discernement s'applique, de préférence, aux exercitants qui sont, par définition, inexpérimentés et, en général, aux personnes qui ne sont pas habituées à examiner leurs propres mouvements intérieurs (mais, comme tous les Exercices, elle peut être adaptée à "chaque type de personne»). En fait, elle fournit des critères de portée générale pour aider l'exercitant «à sentir et à reconnaître, de quelque façon, les différents mouvements qui se produisent dans l'âme» (Loyola 1969, $\mathbb{S} 313$ ), selon le double objectif de le rendre conscient des «esprits» qui l'agitent intérieurement et d'enfin le mettre dans la condition d'agir de façon autonome par rapport à ceux-ci, plus précisément, "pour accueillir les bons et repousser les mauvais" (Loyola 1969, $\mathbb{S} 313$ ). De cette façon, Ignace stabilisait dès le début la distinction fondamentale, basée sur la différence entre esprit/les bons/les (Dieu et les anges) et esprit/les mauvais/les (Satan, «l'ennemi de la nature humaine ", et les démons ou anges mauvais). Il en résulte que du fait de leur simplicité et leur clarté, les règles de la première série fournissent les instruments de base du discernement, puis, dans la seconde série, elles seront ultérieurement précisées et affinées.

Quelques concepts apparaissent de particulière importance et se retrouvent différemment modulés dans les deux séries de règles, généralement combinées avec une attention pour le «temps ", c'est-à-dire pour les circonstances pratiques et les différentes étapes du parcours spirituel de l'exercitant. À cet égard, les deux premières règles sont centrées sur la différence oppositive entre l'action de l'esprit bon et celle de l'esprit mauvais : ceux-ci agissent «au contraire » l'un de l'autre. À ceux qui "passent de péché mortel en péché mortel », l'esprit mauvais «fait imaginer plaisirs et pensées sensuelles » pour les faire persister dans leurs inclinations, alors que l'esprit bon, à l' "opposé ", aiguise et ronge leurs consciences (première règle). La seconde règle, à l'inverse, stipule que pour ceux qui progressent 
dans la purification des péchés (comme, vraisemblablement, l'exercitant de la première Semaine), c'est l'esprit mauvais qui induit les remords et la tristesse, dressant des obstacles sur le chemin du repentir, alors que l'esprit bon donne courage, force, paix et inspirations pour poursuivre le travail ${ }^{18}$. À ce principe du «contraire » s'ajoute, avec la troisième règle, un concept fondamental, celui de la "consolation» (avec son contraire: la "désolation»). La consolation était décrite d'une manière très générale comme «l'état dans lequel se produit dans l'âme un mouvement intérieur pour lequel l'âme demeure enflammée d'amour pour son Créateur et Seigneur, et quand elle ne peut plus aimer pour elle-même aucune autre chose créée sur la face de la terre, mais seulement dans le Créateur de toutes les choses » $(\text { Loyola } 1969, \mathbb{S} 316)^{19}$. Ensuite, Ignace ajoutait que devaient être considérées comme consolation, par exemple, les larmes, quand elles "portent à l'amour de son Seigneur ou à la douleur de ses péchés ", ou encore, si elles dérivent de la «passion du Christ» ou d'autres choses en général «directement ordonnées au [...] service et à la louange» de Dieu. Parmi les consolations, étaient également à inclure «chaque augmentation d'espérance, de foi et de charité, et chaque félicité intime qui appelle et attire aux choses célestes et au salut de son âme». Ensuite, Loyola décrivait, par opposition, l'état intérieur de "désolation " qui, précisément, selon la quatrième règle (Loyola $1969, \mathbb{S} 317)^{20}$, est « tout le contraire de la consolation" et se configure comme l'«obscurité de l'âme, trouble intérieur, l'attirance vers ce qui est vil et terrestre, inquiétude devant des agitations et des tentatives différentes ". De la sorte, alors que la consolation se caractérise comme étant un stimulant et un appel à progresser dans le parcours de conversion, la désolation, au contraire, "pousse à perdre confiance ", à rester "sans espérance, sans amour ", et «l'âme se retrouve tout engourdie, tiède et triste, comme séparée de son Créateur et Seigneur». Et cela,

18. Sans pouvoir développer le problème dans ces pages, il est cependant facile de constater que ces deux premières règles du discernement trouvent des parallèles précis dans l'Autobiographie de Loyola: plus précisément, la première règle correspond à l'expérience de l' «alternance des esprits » expérimentée par Ignace durant sa convalescence dans la maison familiale, des suites de la blessure guérie à Pampelune (Loyola 1943, \$6-8), alors que la seconde règle peut être rapportée soit à la même période par les aspects négatifs, les doutes et les obstacles interposés par l' "esprit mauvais ", soit à certains épisodes remontant à la période de Manresa (voir, par exemple, ivi, $\mathbb{S} 20$, 21,24 , etc.).

19. Ainsi que pour les citations qui suivent.

20. Et pour toutes les citations suivantes. 
puisque les pensées (et les émotions, les états d'âme) qui "naissent de la désolation » sont "à l'opposé » des pensées qui naissent de la consolation.

Les règles cinq et six affrontent le problème de l'attitude pratique à assumer durant le temps de la désolation, selon une perspective double - et opposée, encore une fois. Avant tout, souligne Loyola, il faut «en temps de désolation, ne jamais faire de changements» (Loyola 1969, $\mathbb{S}$ $318)^{21}$ par rapport aux résolutions et aux décisions prises précédemment, et cela parce que si «dans la consolation c'est surtout l'esprit bon qui nous guide et nous conseille, dans la désolation c'est le mauvais ». Et c'est justement pour cela qu'il est nécessaire de ne pas faire de changements: les conseils de l'esprit mauvais «ne peuvent pas nous faire prendre le chemin juste». Toutefois, si c'est une erreur de changer ses décisions dans le temps de la désolation, au contraire, c'est "excellent de se changer vigoureusement» (Loyola 1969, $\mathbb{S} 319$ ) 22 , agissant «contre la désolation même », se dédiant avec une plus grande implication à la prière, à la méditation et à toutes les pratiques pieuses et pénitentielles De plus, la septième règle établissait un principe qui, dans les intentions d'Ignace, devait servir comme une sorte de ressource intérieure ultime, de dernier "point d'appui », pour l'exercitant soumis aux rigueurs de la désolation: précisément parce que la désolation est par définition le temps de l' "épreuve", de la tribulation, Loyola précisait que Dieu laisse "à ses puissances naturelles" (Loyola $1969, \mathbb{3 2 0})^{23}$ celui qui est soumis à la désolation, lui retirant tout autre support — c'est-à-dire: toute sorte de consolation. L'être humain doit faire face "aux différentes agitations et tentations de l'ennemi" seulement à partir de ses propres ressources et sans pouvoir compter sur des aides spéciales de la grâce. Toutefois, cela ne signifie pas qu'il soit totalement privé du «secours divin", même "s'il ne le ressent pas clairement", parce que Dieu "laisse toujours la grâce suffisante pour le salut éternel». Après la huitième règle qui établissait que la patience est la vertu à laquelle doit s'en remettre celui qui se trouve dans la désolation, la neuvième règle (Loyola $1969, \mathbb{S} 322$ ) précisait les trois raisons de la désolation. La première était la tiédeur dans la vie spirituelle que cause la perte des consolations. La seconde dérivait d'une décision divine, visant précisément à mettre à l'épreuve les intentions et les décisions de l'exercitant (et de chaque croyant engagé sur le chemin de la conversion) en absence des consolations. Enfin,

21. Et pour toutes les citations suivantes.

22. Et pour toutes les citations suivantes.

23. Et pour toutes les citations suivantes. 
la troisième raison mettait en lumière le sens ultime de la désolation: précisément à travers la privation de celles-ci, il faut comprendre comment les consolations sont le produit exclusif de la grâce divine et ne dépendent d'aucun acte subjectif. La désolation apparaît comme l'indispensable instrument qui met à nu la vanité de l'individu et chaque tentative d'appropriation personnelle et individuelle des bénéfices spirituels qui sont, de toute façon, toujours dons divins et non produits ou résultats d'un effort subjectif.

Les dixième et onzième règles retournaient sur la consolation, avec comme conseil, pour celui qui se trouve dans ce temps ou cet état intérieur, de "penser à la façon qu'il se conduira dans la désolation à venir », tirant de la consolation même de "nouvelles forces " pour affronter la prochaine désolation ${ }^{24}$ (Loyola 1969, $\mathbb{\$} 323$ ). Par conséquent, au temps de la consolation, il faut s'efforcer de "s'humilier et de s'abaisser » autant que faire se peut, "pensant au peu» qu'on vaut «au temps de la désolation» quand on s'y retrouve "sans cette grâce ou consolation ». À l'inverse, qui se trouve dans la désolation doit se faire l'âme avec l'attitude opposée, pensant "qu'il peut beaucoup avec la grâce suffisante pour résister à tous les ennemis », prenant de Dieu la force qui lui manque ${ }^{25}$ (Loyola 1969, $\mathbb{S} 324$ ). L'importance de la force et de la détermination avec lesquelles s'affronte la désolation était réitérée dans la douzième règle qui recommandait de réagir aux tentations de la désolation avec grande fermeté, "faisant d'une manière diamétralement opposée " aux inspirations de l'ennemi. Les deux règles $^{26}$ (Loyola 1969, $\mathbb{S} 326,327$ ) successives, lesquelles concluaient la première série des règles du discernement exposée dans les Exercices spirituels, développent deux parallélismes ${ }^{27}$ ultérieurs, soulignant que, d'un côté, «l'ennemi se comporte comme un faux amant " qui désire demeurer secret et ne pas être découvert (et donc, souffre et s'échappe lorsque ses tromperies sont révélées au confesseur ou au père spirituel), et, de l'autre côté, qu'il agit "comme un chef militaire" qui, après avoir inspecté le camp adverse, l'attaque à sa partie la plus faible — ainsi, affirme Loyola, l'ennemi inspecte toutes les vertus, cardinales, théologales et morales de l'individu pour ensuite déclencher son attaque sur le côté le plus fragile.

24. Dixième règle. Et pour les citations qui suivent.

25. Onzième règle. Et pour les citations qui suivent.

26. Pour toutes les citations.

27. La douzième règle était construite sur le critère que «l'ennemi se comporte comme une femme ", se faisant "faible devant la force et fort lorsqu'on le laisse faire " (Loyola 1969, $\mathbb{3} 325$ ). 
Bien qu'elles soient disposées l'une après l'autre, les deux séries de règles du discernement - comme il a déjà été discuté — se réfèrent à des moments différents, tant du parcours à étapes des Exercices ignaciens (l'une était en effet relative à la première Semaine, l'autre à la seconde Semaine) que du chemin de conversion intérieur de l'exercitant (ou du pénitent/ croyant en général) et dans les intentions de Loyola, il fallait être prudent dans leur utilisation, puisque si exposées ou appliquées d'une manière incorrecte en relation au temps ou à l'état intérieur de l'individu, elles pouvaient produire des effets négatifs. Ces importantes considérations ont été placées au début des Exercices, par Loyola, dans les abondantes "notes » d'ouverture destinées exclusivement au directeur des exercices. Ainsi, dans les «notes» sept et huit ${ }^{28}$ (Loyola 1969, $\mathbb{S} 7,8$ ), Ignace exhortait - toujours, naturellement, sur la base de sa propre expérience religieuse - le directeur des exercices à «révéler les astuces de l'ennemi» à l'exercitant "désolé et tenté", mais également visité des consolations, et donc à "lui présenter" de la façon opportune "les règles de la première et seconde Semaine, qui servent à connaître les esprits ". Toutefois, dans la note 9, Loyola $(1969, \mathbb{S} 9)^{29}$ précisait clairement que si l'exercitant était engagé dans la première Semaine (avec les méditations sur les péchés, l'enfer, etc.) et que, surtout, s'il n'était pas "déjà versé dans les choses de l'esprit ", il était impératif que le directeur «ne lui parle pas des règles de la seconde Semaine ». Et cela parce que, si la première série de règles «était utile» à qui amorce le parcours de conversion, à l'opposé, "les règles de la seconde [Semaine] le mettraient à mal, traitant une matière trop délicate et trop haute pour qu'il la puisse comprendre ". Dans la fondamentale note 10, Ignace ajoutait d'autres détails. Avant tout, il clarifiait que lorsque l'exercitant «est assailli et tenté sous apparence de bien, c'est le moment de lui parler des règles de la seconde Semaine » (Loyola 1969, $\mathbb{\$} 10)^{30}$. Il poursuivait, ajoutant une précision de grande importance et dense d'implications:

[...] car généralement l'ennemi de la nature humaine tente davantage sous apparence de bien lorsque quelqu'un s'exerce dans la vie illuminative, qui correspond aux exercices de la deuxième Semaine, et moins dans la vie purgative, qui correspond aux exercices de la première Semaine ${ }^{31}$.

28. De même pour toutes les citations qui suivent.

29. Pour toutes les citations suivantes.

30. Ainsi que pour toutes les citations suivantes.

31. Les citations en retrait du texte sont toutes reprises de la traduction des Écrits de Loyola par Maurice Giuliani dans la collection Christus, 1991 (N.D.T.). 
Ignace faisait alors référence (d'une façon tout à fait exceptionnelle) à la conception classique des "trois voies" de la vie spirituelle, exposée surtout par des maîtres et des théologiens mystiques médiévaux, qui faisaient correspondre les étapes essentielles du parcours spirituel à 1) la voie (ou vie) purgative, typique des débutants; 2) la vie illuminative, propre aux «progressants »; et enfin 3) la vie unitive, réservée aux "parfaits». Avec cette brève allusion, Loyola ouvrait ainsi un important interstice sur le chemin «ascendant» des Exercices, dans lesquels la première Semaine remplissait la fonction de la voie purgative (comme le démontrent les exercices dédiés aux méditations sur les péchés desquels l'exercitant doit effectivement se purifier), et la seconde Semaine correspondait, à l'inverse, à la voie illuminative dans laquelle le pécheur, désormais libre des péchés passés, commençait à recevoir les illuminations de la grâce qui le guident et l'orientent dans le progrès de la vie spirituelle. Ignace ne spécifiait pas quelles parties des Exercices représentaient la voie unitive, mais ce n'est pas difficile de supposer que c'est surtout la quatrième et dernière Semaine — culminant dans la célèbre "contemplation pour éveiller l'amour ", dans laquelle l'exercitant devient, sur les traces de saint Paul, un «temple de

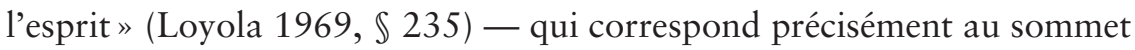
de l'expérience religieuse spiritualiste contenue dans la "voie unitive».

Toutefois, dans la perspective pratique du discernement spirituel, la note 10 établissait un critère essentiel, c'est-à-dire que — précisément parce que le chemin de l'exercitant était désormais en rapport avec la voie illuminative, à savoir en relation avec un stade non plus initial de l'expérience religieuse - l' "ennemi » lance ses assauts «davantage sous les apparences du bien » que du mal: c'est-à-dire se transfigure en un "ange de lumière » et il devient beaucoup plus difficile, tant pour le directeur des exercices (ou pour le père spirituel), que pour l'exercitant (ou le croyant en général), d'en démasquer les tromperies et les tentations. On comprend mieux pourquoi Loyola avait, à peine précédemment, affirmé que les règles du discernement de la seconde Semaine traitent "une matière trop délicate et trop haute » pour ceux qui sont encore engagés dans la voie purgative (la première Semaine), caractérisée en général par des «tentations grossières et évidentes» (Loyola 1969, $\mathbb{~ 9 ) . ~}$

La seconde série de règles, plus brève que la première (huit règles dont deux sont consacrées à la "consolation sans cause » - contre 14), aborde donc les problèmes plus subtils et délicats qui surgissent dans la voie illuminative, le stade du progrès qui suit la conversion et le détachement des péchés. On y traite, comme il est évident, d'une phase décisive et qui 
peut durer très longtemps (au-delà de la «synchronisation » des Exercices), dans laquelle l'exercitant (et le croyant en général) est, dans un certain sens, "en équilibre instable", suspendu entre le risque des rechutes dans la vieille vie et l'espérance et le désir de parvenir à un contact ou à une communication plus directe avec Dieu en accédant à la voie unitive. Toutefois, comme il a déjà été mentionné, dans la perspective d'Ignace, la condition actuelle de l'exercitant est opposée, en tant qu'état ou «temps » intérieur, à celle du pénitent qui amorce le chemin de conversion. À présent, «Dieu et ses anges» stimulent l'exercitant non pas avec l'âpre et amer aiguillon du remords, mais en lui procurant des "motions» (mouvements, sensations, émotions, pensées) intérieures caractérisées par la joie spirituelle et l'allégresse qui chassent les troubles et les tristesses, inspirés plutôt par l' «ennemi ». Au contraire, Satan et ses anges «luttent contre cette allégresse et consolation spirituelle ", affirme Loyola, en "proposant » à l'âme des «raisons apparentes, subtilités et sophismes perpétuels ${ }^{32}$ » (Loyola 1969, $\mathbb{S}$ 329). Sans pouvoir ici s'arrêter sur l'angéologie ignacienne, c'est-à-dire sur le rôle central qu'Ignace attribuait, dans la seconde Semaine, aux anges, bons ou mauvais, disons simplement que de la première règle émerge le problème central du discernement des «progressants »: le noyau essentiel constitué des "pensées ". Ainsi, après avoir introduit le thème de la "consolation sans cause ${ }^{33}$ » (Loyola 1969, $\$ 330$ ) (sur lequel on reviendra), Loyola s'occupait des nombreuses nuances et caractéristiques dérivant de la "consolation avec cause ", la consolation qui dérive de quelque "sentiment ou connaissance préalable de quelque objet» ou contenu de l'intellect ou de la volonté (Loyola 1969, $\mathbb{S} 330$ ). D'abord, la troisième règle établissait que tant l'ange bon que l'ange mauvais «peuvent consoler l'âme, mais à des fins opposées ${ }^{34}$ » (Loyola 1969, $\mathbb{3} 331$ ). Donc — et il s'agit là d'un point très délicat qui modifiait ce qui avait été affirmé dans la première série de règles (où la consolation était toujours le produit de l'«esprit bon») —, pour l'exercitant engagé dans la voie illuminative, la consolation peut également être causée par l'ange mauvais, qui "au contraire de l'ange bon» (qui se sert d'elle "pour le progrès de l'âme»), l'utilise pour attirer l'exercitant vers "ses buts malveillants et sa perversité». Donc, il apparaît particulièrement important d'identifier des critères pour distinguer quand la

32. Première règle.

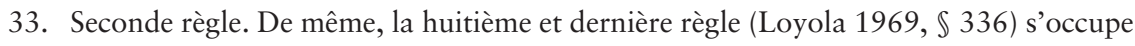
du problème de la consolation sans cause.

34. Pour cette citation et les suivantes. 
consolation dérive de l'ange mauvais et quand elle dérive du bon. Les quatre règles successives fournissent les indications et les fondements essentiels à ce but ${ }^{35}$. La quatrième règle de la seconde Semaine explicite le thème fondamental, celui de l'ange mauvais qui, "lorsqu'il se transforme dans l'ange de la lumière ${ }^{36}$ » (Loyola 1969, $\mathbb{S} 332$ ), prenant les «apparences du bien ", s'efforce de "s'introduire dans les sens de l'âme fidèle pour enfin la conduire avec lui ». Son procédé consiste à insinuer "pensées bonnes et saintes, en consonance avec l'âme juste, et puis [...] chercher de la porter à ses fins [...], à ses tromperies secrètes et intentions perverses ». Précisément parce que l'ange mauvais, sous l'apparence de l'ange de lumière, «insinue » surtout des "pensées ", la règle cinq établit qu' «il faut prêter une grande attention au cours de nos pensées ». Par la suite, Ignace spécifiait ce principe fondamental centré sur l'attention au "cours des pensées ", précisant que "si le début, le milieu et la fin sont complètement bons, ceci est un signe de l'ange bon ». À l'inverse, "si le cours de nos pensées nous conduit à la fin à quelque chose de mauvais, de futile, ou de moins bon que ce que l'âme projetait au début ", alors "cela est un signe clair que c'est l'esprit mauvais qui procède». Selon l'expérience d'Ignace, il en va de même si le "cours des pensées" conduit, à la fin, à quelque chose "qui affaiblit, inquiète et trouble l'âme lui retirant la paix, la tranquillité et le repos qu'elle avait avant». Avec beaucoup de raffinement psychologique, la sixième règle précise qu'une fois "découvert et reconnu l'ennemi [...] par sa queue de serpent et par la fin mauvaise qu'il inspire », il est particulièrement "utile, à celui qui a été tenté, d'immédiatement observer le cours des pensées bonnes ", sous lesquelles, précisément, s'est introduit l' "ennemi » sous la forme de l' "ange de lumière ", "et leur point de départ: comment peu à peu, il tâcha de nous faire abandonner la suavité et la joie spirituelle [...] jusqu'à nous conduire à ses intentions dépravées ». Enfin, Loyola concluait son enseignement en observant: "ainsi, grâce à cette expérience reconnue et annotée, à l'avenir, on se préservera de ses tromperies habituelles ». C'est un des passages des Exercices spirituels dans lequel se révèle, explicitement, la vision d'Ignace: rendre conscient et enfin, expert et autonome, l'exerci-

35. Sans pouvoir approfondir le problème, il convient d'au moins observer que de même dans ce cas, il serait possible de tracer de nombreux parallèles entre cette seconde série de règles et plusieurs passages de l'autobiographie de saint Ignace, montrant comment les règles de la seconde série dérivent aussi, et surtout, de son expérience religieuse personnelle.

36. Pour cette citation et les successives, à l'exception de celle qui vient immédiatement après qui est tirée de Loyola $(1969, \mathbb{\$} 10)$. 
tant, et le croyant en général, face aux «esprits» qui le meuvent, afin qu'il sache par lui-même discerner la manifestation de la volonté de Dieu dans le complexe langage de l'âme. La dernière règle de la seconde série (à l'exception de la suivante, la huitième, dédiée de nouveau à la consolation sans cause) concernant la voie illuminative et les consolations avec cause, fait voir, très clairement, comment la matrice originaire des règles mêmes ne fut rien d'autre que l'expérience religieuse personnelle de Loyola. En effet, la septième règle indique une série de distinctions entre l'action, dans l'âme, de l'ange bon et de l'ange mauvais qui ne font pas référence à autre chose qu'une expérience directe et subjective qu'Ignace s'efforçait de transmettre et d'enseigner :

À ceux qui procèdent de mieux en mieux, l'ange bon touche l'âme avec douceur, paisiblement et suavement, comme la goutte d'eau qui entre dans une éponge; alors que le mauvais la touche cruellement, avec violence et agitation, comme la goutte d'eau qui tombe sur la pierre. À ceux qui procèdent de mal en pis, les mêmes esprits touchent d'une manière opposée, la raison de cela étant dans la disposition de l'âme, à être contraire ou semblable aux anges: en effet, lorsque contraire à ceux-ci, leur entrée est bruyante et sensible, perceptiblement; lorsque semblable, l'entrée est silencieuse, comme dans sa propre maison à porte ouverte. (Loyola 1969, $\mathbb{\$} 335$ )

\section{Discernement et décision: la méthode de l'élection}

Cependant, pour comprendre le procédé global du discernement ignacien, il faut encore s'arrêter sur la fonction du binôme consolation/désolation et ensuite observer comment ce couple d'opposition (également, bien sûr, avec les deux séries de règles du discernement) interagit avec les procédures d'élection. Les deux mouvements intérieurs de consolation/désolation constituent de véritables états d'âme qui reflètent la nature de l'esprit agissant à un moment donné sur la psychologie de l'exercitant. Alors qu'il décrit son état d'âme au directeur spirituel, non seulement l'exercitant lui communique ses propres conditions intérieures, mais il le met en mesure d'intervenir avec des conseils opportuns pour faire face à la désolation ou pour mieux orienter la consolation vers "la fin pour laquelle l'homme est créé ", l'adoration et le service de Dieu, selon le "Principe et fondement" de tous les Exercices ${ }^{37}$ (Loyola 1969, $\mathbb{S} 23$ ). De plus, le présupposé établi

37. Sur la signification et l'importance du «Principe et fondement », voir Mongini (2011, 145-151). 
par Loyola, selon lequel l'esprit bon est directement connecté à l'action divine $^{38}$ (Loyola 1969, $\mathbb{S} 329$ ), implique que son effet, la consolation, semble dans un rapport étroit avec la recherche de la volonté de Dieu "chercher et trouver la volonté de Dieu dans l'organisation de sa propre vie ", comme l'exprimait Loyola — qui est le but de tous les Exercices (en tant que condition nécessaire à l'accomplissement de la "fin de l'homme ", précisément l'adoration et le service). En effet, selon la perspective d'Ignace, la consolation, bien qu'ayant une valeur intrinsèque, constitue surtout en tant qu'expression de l'esprit bon d'origine divine — le symptôme, le signe par excellence de la manifestation positive de la volonté de Dieu, de son approbation (et cela est vrai aussi en ce qui concerne la seconde Semaine et la correspondante seconde série de règles du discernement, centrée sur la nécessité de distinguer la consolation authentique de la fausse, induite de l'ange mauvais). Cela signifie que, dans le cadre des Exercices, c'est l'entière psychologie de l'exercitant, sa conscience (et à bien voir, son corps même en ce qu'il est le lieu de manifestation des sensations et des émotions) à fonctionner comme une sorte de camp fermé dans lequel se manifeste la volonté de Dieu, qui se reflète justement dans les différents états d'âme en rapport avec la consolation. L'ensemble des règles du discernement et leur procédé articulé constituent, comme il en a déjà été mention, la clé pour analyser et identifier, dans l'expérience intérieure concrète de l'exercitant, comprise dans la perspective d'une succession d'états d'âme, les indications de la volonté divine "dans l'organisation de sa vie ». La psychologie de l'exercitant — davantage si elle est entraînée et aiguisée dans la pratique des exercices variés des différentes Semaines - devient ainsi une sorte d'hypersensible sismographe en mesure d'enregistrer les plus minimes mouvements psychologiques, les plus imperceptibles motions intérieures - indices précieux de la grâce - qui deviennent,

38. Il importe de bien saisir ce point, sans tomber dans l'équivoque d'attribuer à Loyola une sorte de manichéisme trompeur. D’une manière générale, même l' «esprit mauvais » est soumis à la «volonté de Dieu »; mais il s'exprime en rapport avec l'exercitant, à savoir sur un plan spécifique et délimité (d'une manière encore plus médiatisée et indirecte de la "consolation" qui procède de l' "esprit bon"), et, assumant les formes de la «tentation", de l' "aridité", de la «difficulté à trouver Dieu ", de la "distraction», configure le temps de l' «épreuve» (de la «tribulation », selon un langage typique de la spiritualité jésuite successive, mais qui trouve déjà ses racines chez Lainez et Ribadeneira, outre Loyola), c'est-à-dire de l' "examen » divin des intentions et des dispositions de l'exercitant; dans le cadre de l'élection, il exécute la fonction de «confirmation négative ", exprimant, d'une certaine manière, la désapprobation divine du choix et offrant ainsi l'opportunité de le refaire, de le réorienter. 
dans l'analyse rationnelle, autant de signes de la volonté divine jusqu'à composer un véritable langage à travers lequel s'exprime la divinité. De la sorte, l'expression de la volonté divine retrouve dans le binôme opposé consolation/désolation, chacune étant articulée et modulée dans une vaste gamme de nuances émotives et psychologiques, son instrument essentiel de communication avec l'être humain. Par conséquent, et d'un point de vue général, on peut comprendre comment le discernement dans les Exercices spirituels de Loyola se configure comme la véritable méthode pour déchiffrer le langage particulier de Dieu dans l'âme, pour interpréter et entendre la langue avec laquelle la divinité parle à l'homme.

L'idée que l'ensemble des procédés articulés par Loyola dans les Exercices spirituels finisse par donner lieu à une véritable «langue», à un "système linguistique ", a été proposée principalement par Roland Barthes dans un essai bien connu (Barthes 1977, 29-64). Toutefois, sous cet aspect, la perspective de lecture avancée par Barthes apparaît exposée au risque de l'équivoque ou, pour le moins, d'un certain réductionnisme. S'il est certainement approprié de soutenir que Loyola a voulu construire un système d' «interlocution» avec la divinité, une «langue de l'interrogation» de Dieu, il est nécessaire d'avoir à l'esprit que l'aspect central de cette interlocution (excluant, bien entendu, les «illuminations » directes) est constitué de «signes » psychologiques, et à la limite «psychiques », dans le sens que ce sont les différents états d'âme - avec toutes leurs nuances, natures et origines — aptes à représenter les "signes" et les "termes ", à savoir les "mots» les plus caractéristiques de ce "langage». Par conséquent, il n'existe pas, dans ce "système linguistique ", de véritables espaces pour le "silence de Dieu" ${ }^{39}$ " (Barthes 1977, 64), parce que cela équivaudrait à une absence de manifestation de la volonté divine, avec une seule exception: tel silence peut se produire seulement dans le cas d'une alternative égale, c'est-à-dire "indifférente ", ce qui est une alternative apparente, qui ne nécessite pas d'intervention divine en ce qu'elle ne requiert pas un "choix" ni une interprétation.

Il est nécessaire, bien qu'on ne puisse ici s'y attarder, d'au moins mentionner un important aspect qui concerne le rapport entre ce que Barthes a défini comme le «code de la demande» et le "code de la réponse ». Non sans raison, il ramenait les Exercices ignaciens à un problème plus général, celui de la "mantique, art de la consultation divine", soulignant que cet art antique "comprend deux codes: celui de la question adressée par

39. Cela ne doit pas être confondu avec le différé de la réponse, ou «le retard du signe». 
l'homme à la divinité, celui de la réponse renvoyée par la divinité à l'homme $^{40} »$ (Barthes 1977, 35). Toutefois, dans sa perspective, le premier code, celui de la demande, "se trouve principalement dans les Exercices, le second (ou code de la réponse) dans le Journal» autobiographique de Loyola ${ }^{41}$. Il convient de relever que Barthes ne saisit pas ou comprend mal un point capital: le Journal spirituel d'Ignace n'est pas un "code» de la réponse de la divinité à l'interrogation de l'homme, mais la transcription d'un exemple de réponse de la divinité - celle qui a été faite à Ignace. Le code de la réponse est en fait un autre: ce sont précisément les règles du discernement, sur lesquelles on s'est arrêté précédemment, le véritable «code de la réponse de Dieu » dans l'âme de l'exercitant, la fondamentale «syntaxe», et ensemble la «rhétorique», de la «langue divine» qui s'inscrit chez l'exercitant. En effet, le Journal spirituel de Loyola constitue un extraordinaire exemple d'application des règles du discernement et de l'élection à un cas particulier. Et sur le plan historique et biographique, il montre de quelle façon - dans une période donnée et sur une problématique spécifique - le fondateur de la Compagnie de Jésus utilisait la méthode de l'élection enseignée dans les Exercices comme instrument de résolution de questions de particulières importances. En outre, ce fait montre comment sont dans l'erreur ceux qui interprètent l'élection comme une pratique à effectuer une seule fois dans la vie ou dans un nombre restreint de cas exceptionnels, toujours concernant son propre «état de vie »: l'expérience de Loyola montre le contraire, c'est-à-dire que l'élection est applicable à toute interrogation et tout choix qui revêtent une quelconque importance pour l'individu. Donc, le fait d'avoir mal compris et sous-évalué la signification et la fonction des règles du discernement constitue une des limites de fond du point de vue de Barthes. Cependant, pour mieux comprendre le rôle fondamental joué par les règles du discernement dans l'élection, dans le choix, il faut s'arrêter sur la méthode exposée par Ignace dans les Exercices. Tout ce qui a été dit jusqu'à présent trouve une exemplification achevée dans la conception de l'élection telle qu'exposée par Loyola, dans laquelle les différents degrés, niveaux et modalités de l'expérience religieuse des Exercices mènent à une synthèse d'exceptionnelle densité.

40. Pour toutes les citations, de même les suivantes.

41. Le même concept (Barthes 1977, 33 et 63) où il parle du Journal comme «ébauche du code divin ». Pour le texte, en traduction, du Journal d'Ignace, on se reportera spécialement à Loyola (2007). 
Ignace distinguait "tres tiempos", trois moments ou modalités, "pour faire un sain et bon choix ${ }^{42} »($ Loyola $1969, \mathbb{S} 175-177)$ :

Le premier temps est quand Dieu notre Seigneur meut et attire la volonté d'une telle façon que, sans douter ni pouvoir douter, l'âme fidèle suit ce qui lui est indiqué, c'est ce que firent Saint Paul et Saint Matthieu, en suivant le Christ notre Seigneur.

Le deuxième: quand on reçoit suffisamment de lumière et de connaissance par l'expérience des consolations et des désolations, et par l'expérience du discernement des esprits.

Le troisième temps est tranquille: considérant d'abord pourquoi l'homme est né, c'est-à-dire pour louer Dieu notre Seigneur et sauver son âme, et désirant cela, on choisit, comme moyen, une vie ou un état qui se situe à l'intérieur de l'Église, afin d'y trouver une aide pour le service de son Seigneur et le salut de son âme.

J'ai dit un temps tranquille: quand l'âme n'est pas agitée par divers esprits et use de ses facultés naturelles, librement et tranquillement.

On a déjà dit comment l'élection consiste en une alternative (par exemple: contracter le mariage ou pas; embrasser le statut religieux ou pas; accepter un bénéfice ou pas, etc.) qui requiert une décision, laquelle, à son tour, est susceptible de modifier en tout ou en partie le statut de vie de l'exercitant. Comme il est possible de voir, les trois moments de l'élection correspondent à autant de niveaux de certitude, qui à leur tour impliquent plusieurs aspects de la psychologie individuelle et sont en rapport à différentes typologies d'expérience intérieure.

Selon un procédé à l'œuvre tout au long des Exercices, Loyola dissimule sous des expressions à l'apparence insignifiante des contenus plus radicaux et proprement spiritualistes de l'expérience religieuse ${ }^{43}$; formulations qu'il convient de patiemment décrypter. C'est d'ailleurs le cas du «premier temps» de l'élection auquel correspond le degré maximal de certitude intérieure. À l'apparence anodine, l'exemple cité par Ignace et relatif à Paul et à Matthieu — tous les deux apôtres, ce qui témoigne de la centralité du modèle apostolique et du thème de l'Église primitive dans la pensée religieuse du fondateur de la Compagnie de Jésus — fait allusion,

42. Pour toutes les citations.

43. Un exemple de particulière importance est donné, sans qu'on ne puisse approfondir le problème ici, de la «contemplation pour parvenir à l'amour ", plusieurs fois mentionnée (Loyola 1969, \$230-237). 
en réalité, à un événement bien précis : il s'agit ici du véritable appel effectué par le Christ qui demande à ses disciples de le suivre (Mt 9,9; Ac 9,119). La manifestation de la volonté divine est à son degré maximal d'évidence, auquel correspond l'absolue certitude et la connaissance de sa volonté de la part du sujet, qui non seulement ne doute pas, mais «ne peut douter », tant est ici explicite la volonté divine et, conséquemment, le fidèle prêt à consentir.

Cependant, il est fondamental de souligner que l'appel des deux apôtres est survenu selon des modalités très différentes: dans le cas de Matthieu, lors d'une rencontre physique, historique, avec le Christ qui l'aperçoit et lui demande de le suivre; ce qui est frappant est la soudaineté, l'immédiateté avec laquelle Matthieu répond: il se lève et suit le Christ, avec obédience et promptitude absolue. Le cas de Paul est très différent. En effet, "l'apôtre des Gentils» n'a jamais rencontré physiquement le Christ et la modalité de l'appel est tout à fait différente. Le Christ lui apparaît dans une vision de lumière éblouissante, l'apostrophe et en provoque la conversion ${ }^{44}$. L'appel de Paul est de type extatique, visionnaire et, tout en étant historique, s'explique selon une modalité intemporelle, métahistorique, non conditionnée par la localisation physique de la rencontre. Pourtant, puisqu'Ignace la place sur le même plan que celle de Matthieu, cela ne peut que signifier que le degré de certitude absolue s'avère le même: rencontrer le Christ dans une vision est comme le rencontrer en Palestine, dans les rues de Capharnaüm. De la sorte, Loyola laissait prudemment, mais avec fermeté, entendre que le "premier moment " de l'élection coïncide avec l'illumination intérieure, avec une expérience religieuse radicalement spiritualiste et subjective dans laquelle se manifeste directement la volonté de Dieu, précisément à la façon de saint Paul: modèle et archétype de la certitude qui provient de l'illumination, la même certitude sur laquelle fondait sa légitimation la mission apostolique de Paul. Encore une fois, c'était le "treizième apôtre " le véritable, authentique modèle du fondateur de la Compagnie de Jésus: le modèle universel, dans un certain sens, de ceux qui, bien que n'ayant jamais rencontré le Christ dans l'histoire, le rencontrent en esprit, tirant de cette source leurs indubitables certitudes. De cette manière, au sommet des "trois moments pour faire un choix", Ignace plaçait l'illumination intérieure qui provoque l'assentiment immédiat de la volonté et fait tel qu' « une âme pieuse suit ce qui lui est montré » dans l'évidence de la vision intérieure.

44. Voir l'épître aux Galates, 1,11-17, et surtout les Actes des Apôtres, 9,1-19. 
Le caractère exceptionnel, ou mieux, la spécificité du "premier moment" se reflète dans l'absence d'indications psychologiques à son égard dans les Exercices, à l'exception de l'observation qui distingue, pour Loyola, la certitude intérieure: "sans douter ni pouvoir douter ", précisément, face à l'appel direct de Dieu. En principe, dans la perspective ignacienne exposée dans les Exercices spirituels, le "premier moment» de l'élection ne semble pas soumis au discernement. À l'inverse, le «second» et le «troisième temps » correspondent à des expériences religieuses qui se placent à deux niveaux psychologiques différents et inférieurs et qui sont précisément en rapport avec le discernement des esprits.

Centré sur l'«expérience des consolations et des désolations» et sur l' "expérience dans le discerner les différents esprits ", le "second temps» semble particulièrement en rapport avec l'expérience religieuse typique du parcours des Exercices, centrée sur l'alternance du couple consolation/ désolation, duquel on a précédemment discuté. Ici, ce sera surtout l'apparition de la consolation à indiquer, dans l'action de l'esprit bon, de quel côté de l'alternative s'incline, pour ainsi dire, la volonté divine à laquelle l'exercitant devra s'efforcer de conformer son propre choix. Le «second temps » est donc connecté étroitement au discernement et à ses règles (surtout à celles de la seconde Semaine) et constitue une expérience religieuse dont le véritable champ est la psychologie de l'exercitant, entraîné et discipliné au travail des exercices.

Tandis que le «troisième temps » représente un exemple significatif de ce qui peut être défini comme rationalisme ignacien. Sa spécificité provient du fait que ce genre d'élection se produit justement en absence des conditions qui définissent la précédente: la «tranquillité » est exactement l'état d'âme qui "n'est pas agité des différents esprits ", c'est-à-dire des bons et des mauvais et, donc, n'expérimente ni consolation ni désolation. Dans ce cas, le discernement est entièrement lié au raisonnement, à l'entendement qui procède de façon analytique. Ce genre d'élection est non seulement le plus difficile, mais également le moins certain, non pas tant en raison d'une méfiance de la part de Loyola envers les "puissances naturelles ${ }^{45}$ » que parce

45. Les nombreux paragraphes qu'Ignace dédie au «troisième temps » de l'élection (voir Loyola 1969, $\mathbb{S} \mathbb{S} 177-188$ ) montrent non seulement l'importance en lien avec ce spécifique type de choix, mais aussi comment il recourrait à un vaste éventail de tactiques, d'hypothèses, de variations de perspectives, pour consentir à la raison de déployer toutes ses ressources pour filtrer, en mode analytique, tous les aspects possibles de l'alternative, à la lumière d'une "discipline de l'attention » qui est certainement un des résultats - et des méthodes - typiques de la pratique des Exercices. 
que dans ce genre d'expérience intérieure, la voix divine est faible puisque voilée de trop de médiations qui s'interposent entre l'exercitant et la divinité. Si éloigné du laisser "opérer inmediate le Créateur avec la créature " (Loyola 1969, $\mathbb{S 1 5}$ ), qui est une des prémisses essentielles des Exercices et qui trouve sa finalité dans l'amour comme directe "communication entre les deux parties", entre l'homme et Dieu, selon la «contemplation pour parvenir à l'amour» (Loyola 1969, $\mathbb{S} 230-237$ ). De là le recours à l'image de l'«aiguille de la balance» et de l' «indifférence» au regard des contenus du choix, dispositions indispensables pour consentir à la balance intellectuelle de l'exercitant pour percevoir la «grande poussée rationnelle» (faible, extrême, indice de l'action de la volonté divine) qui devrait enfin la faire pencher vers un bord ou l'autre des alternatives. La décision prise, l'exercitant, précise Ignace, devra se recueillir en prière et "offrir » à Dieu ce choix afin que «sa divine majesté veuille l'accueillir et le confirmer»: d'une telle façon, à bien y regarder, le "troisième temps" revient sur le «deuxième » puisque le bas degré de certitude requiert d'ultérieures confirmations du choix de la part de la divinité, à savoir la manifestation de signes qui appartiennent au cadre, soit du "premier temps » (celui illuministe ou d'inspiration directe), soit, plus vraisemblablement, du « second temps ", c'est-à-dire à l'alternance du couple consolation/désolation et à leur discernement.

Les procédures complexes de l'élection et de leur discernement jusqu'ici examinées ne seraient pas complètes sans aborder, même brièvement, un point demeuré à l'arrière-plan. Précédemment, on a mentionné le fait que le «premier temps » de l'élection, relatif à l'inspiration directe de Dieu ou à l'illumination intérieure, selon Loyola, comporte une certitude absolue: «sans douter ni pouvoir douter». Par conséquent, ce genre d'élection ne semble pas soumis au discernement précisément en ce que, par définition, elle est soustraite à la "diversité des esprits » puisqu'en elle agit et se manifeste un seul "esprit » et une seule volonté, la divine, avec une telle clarté, force et évidence, qu'elle rend superflu l'exercice de la discretio. Cette centralité et cette excellence du moment spiritualiste - dans la forme de l'inspiration divine directe ou de l'illumination - dans les Exercices sont assurément le signe de l'expérience religieuse personnelle de saint Ignace et de ses caractéristiques spécifiques, fortement marquées par l'alumbradismo castillan de son temps, dont il sera question sous peu. Cependant, passant du plan théorique et général à la perspective pratique du discernement spirituel, il faut observer que Loyola avait tracé un cadre plus complexe et plus articulé. Dans la seconde série de règles du discernement insérée dans les Exercices, à la seconde règle, Ignace, reprenant le thème de la consola- 
tion, duquel il a déjà été mention, avait inséré un concept très particulier, celui de «consolation sans cause»:

Il appartient à Dieu notre Seigneur, seul, de donner à l'âme une consolation sans cause précédente; car c'est le propre du Créateur d'entrer, de sortir, de produire en elle une motion, l'amenant tout entière à l'amour de sa divine Majesté. Je dis: sans cause, c'est-à-dire sans qu'il y ait de sentiment préalable ou de connaissance de quelque objet par lequel pourrait venir cette consolation par le moyen des actes de l'intelligence et de la volontét ${ }^{46}$. (Loyola

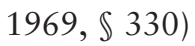

Qu'en principe, la consolation sans cause soit exempte de tromperie et soit, par conséquent, soustraite au discernement, Loyola l'affirmait en ouverture de la huitième règle de la seconde série, règle, elle aussi, dédiée à la consolation sans cause: "Quand la consolation est sans cause il n'y a pas de tromperie en elle, puisque, comme on l'a dit, elle est de Dieu notre

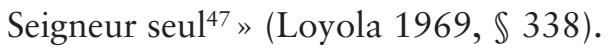

Bien qu'elle ne soit pas explicitée par Ignace, il existe une étroite connexion entre l'expérience spiritualiste que constitue le "premier moment » de l'élection et la consolation sans cause. Tout d'abord, la première est marquée, comme on l'a mentionné, par la certitude, puisque d'elle on ne doute pas et on ne peut douter; toutefois, la seconde présente, elle aussi, la même nature ou le même statut en ce qu'elle "ne comporte pas de tromperie » et donc, tout autant de son intrinsèque vérité, on pourrait dire, on ne doute pas ni ne peut douter. En second lieu, la consolation sans cause, non seulement "vient uniquement de Dieu ", mais elle vient aussi directement de lui, qui peut "entrer, sortir, produire» directement dans l'âme les consolations sans cause et, de cette façon, l'attirer "toute entière dans l'amour de sa divine majesté». De même, dans le "premier temps " de l'élection, "Dieu notre Seigneur stimule et attire la volonté » de l'individu de manière directe. Les deux expériences sont donc similaires. Un autre aspect de cette affinité est que comme le "premier temps» de l'élection est (comme on l'a vu) soustrait au discernement en ce qu'il ne comporte aucun doute ni la possibilité de celui-ci, la consolation sans cause est, elle aussi, soustraite au discernement, en ce que par sa propre nature, il est immédiatement évident qu'elle aussi «vienne uniquement» et directement «de Dieu », et il n'y a donc pas de doute possible quant à l'«esprit »

46. Seconde règle. Sur la consolation sans cause, voir par exemple Gil (1971); Mondel (1977); Gouverneur (1980).

47. Huitième règle. 
qui la produit. De la sorte, il est, en principe, indiscutable qu'Ignace ait assigné au moment spiritualiste et illuministe de l'expérience religieuse un rôle et un statut de relief exceptionnel: celle-ci, et seulement cette typologie de l'expérience religieuse, était, pour Loyola, soustraite à tous les doutes, pourvue de certitude et exempte du scrutin du discernement.

Même si, sur le plan théorique, ce qui a été dit est vrai, dans le délicat passage à la perspective pratique de l'expérience religieuse, Loyola avait toutefois ajouté d'importants correctifs, encore une fois résultant de son expérience illuministe personnelle. Ainsi, la huitième règle du discernement de la seconde Semaine, après avoir affirmé que la consolation sans cause ne comportait pas de tromperie, poursuivait en précisant:

Cependant la personne spirituelle à qui Dieu donne cette consolation doit regarder et discerner avec beaucoup de vigilance et d'attention le temps même de cette consolation présente de celui qui suit, où l'âme reste toute brûlante et favorisée du bienfait reçu et des suites de la consolation précédente. Souvent, en effet, dans ce second temps, soit par son propre raisonnement à partir de liaisons et de déductions nées de nos idées et jugements, soit du fait du bon esprit ou du mauvais, nous concevons divers projets et diverses opinions qui ne sont pas données immédiatement par Dieu notre Seigneur. C'est pourquoi il est nécessaire de les examiner très attentivement avant de leur accorder entier crédit et de les mettre à exécution.

À rapporter, sur le plan pratique, la consolation sans cause - somme et emblème, comme il a été mentionné, de l'expérience religieuse illuministe et spiritualiste de saint Ignace —, ou mieux, de son "second temps ", à la sphère du discernement spirituel, Ignace de Loyola complétait avec beaucoup de raffinement son enseignement sur la discretio, légitimant, au même moment, de façon définitive l'illumination spirituelle et l'inspiration intérieure comme moments décisifs de l'expérience religieuse - en ce qu'elles sont les seules à être absentes de tromperies et immunisées au doute, et les seules à être vraiment génératrices de certitude - et laissant en héritage, aux générations successives, un patrimoine d'expériences religieuses particulier au centre duquel se posait la nécessité incontournable du discernement des esprits.

\section{Le discernement et l'élection comme problème historique: les Exercices spirituels entre hérésie et orthodoxie}

Dans les pages précédentes, on a voulu comprendre, d'une certaine façon iuxta propria principia, tant les mécanismes principaux de fonctionnement 
des règles du discernement et de l'élection dans les Exercices ignaciens, que plusieurs aspects de l'expérience religieuse, ainsi que le révélait le texte luimême dans ses complexes articulations. Voilà pourquoi la véritable dimension historique du célèbre livre de Loyola est demeurée, en grande partie, en arrière-plan. Toutefois, elle apparaît désormais indispensable, davantage si l'on se penche sur l'énorme conditionnement représenté par la tradition interprétative jésuite et en général catholique, qui, bien que contribuant à l'extraordinaire fortune et diffusion des Exercices spirituels, en a également marqué la déformation parallèle d'un point de vue exclusivement pieux (et souvent unie à une perspective d'actualisation pastorale), y dissipant les spécificités historiques et théologiques dans la continuité abstraite d'une tradition de spiritualité au fond anhistorique et atemporel, et finissant par dissimuler sous un épais voile hagiographique et apologétique une partie essentielle de la signification et des implications du texte de Loyola. Une déformation et un appauvrissement, à bien y voir, qui ont parallèlement distingué la reconstruction du profil et du message religieux du fondateur de la Compagnie de Jésus; déformations et appauvrissement auxquels seulement de récentes études ont tenté, avec peine, de s'y soustraire.

Or, dans ces pages, on ne peut faire que quelques références aux nombreux conflits, aux âpres accusations, aux suspicions tenaces qui accompagnèrent les Exercices et leur auteur. Accusations et conflits surgis précocement, dès la moitié des années 1520 , alors que Loyola fut soumis à deux procès de l'Inquisition pour suspicion d'alumbradismo, à Alcalá de Henares et à Salamanque. Ces procès se prolongeront pendant longtemps, notamment à Paris et en Italie. Entre-temps, en 1540, il donnera naissance, avec les socii, à la Compagnie de Jésus. Une ombre d'hétérodoxie, qui ne s'est jamais retirée complètement, s'étend ainsi sur les premiers jésuites et sur l'emblématique protocole de leur proposition religieuse, précisément les Exercices spirituels, dont la rédaction débutée dans l'Espagne marquée par l'enseignement d'Érasme et des doctrines des alumbrados, se conclut, plusieurs années plus tard, après de multiples révisions, dans l'Italie du pape Paul III Farnèse, où l'incessante propagation de la protestation luthérienne s'ajoutait à la crise profonde de l'Église, au pullulement des différentes hérésies dans toutes les couches sociales, aux tentatives irénistes élaborées au sein de l'évangélisme italien de Contarini, mais où, avec la diffusion prudente mais rapide de la pensée de Juan de Valdès ${ }^{48}$, prenait

48. Sur les formes et les contenus de la direction spirituelle de Valdés, voir l'essai de Firpo (2008, 183-220); sur l'hérésie en Italie, voir la dense synthèse de Firpo (1993). 
forme la réponse du parti des «intransigeants» guidé par Gian Pietro Carafa (le futur pape Paul IV), alors qu'était imminente la réorganisation de l'Inquisition de 1542. Donc, sur l'arrière-plan de la grande crise religieuse du Xvi ${ }^{\mathrm{e}}$ siècle italien et européen, il faut également joindre la figure d'Ignace de Loyola, pour entrevoir ainsi le véritable problème historique qui est à la racine des Exercices spirituels: celui de la matrice et des prémisses dont l'expérience du fondateur de la Compagnie de Jésus était issue, et, ensuite, des formes qu'elle prendra dans la mutation rapide des contextes historiques et idéologiques.

En effet, si elle est reconduite à ses origines espagnoles, aux contacts désormais documentés sans ombre de doute - au-delà de ses démentis ambigus - entre Loyola et les milieux et les représentants de l'alumbradismo ${ }^{49}$, son expérience religieuse laisse entrevoir plusieurs traits caractéristiques du spiritualisme radical et de l'illuminisme qui ont distingué le monde hétérogène des hérétiques castillans et les multiples analogies de l'enseignement ignacien d'avec les plus célèbres représentants de cette hérésie redoutée ${ }^{50}$. Sans pouvoir parcourir de façon analytique les nombreuses affinités que la pensée de Loyola (exprimée par exemple dans les autobiographies Acta Patris Ignatii et dans le Journal spirituel) et les Exercices présentent avec les courants les plus anciens de l'alumbradismo, il est toutefois indispensable de s'arrêter, à titre d'exemple, sur un problème particulièrement important, utile pour faire apparaître l'ambiguiité intrinsèque - et les conséquentes contiguïtés historiques avec certains thèmes de l'alumbradismo - d'un des aspects centraux des Exercices: le thème de la communication directe de l'exercitant avec la divinité qui est le fondement de tous les Exercices, "trouver la volonté de Dieu», et de l'élection. Au même moment, ce thème impliquait également les règles du discernement en tant que règles qui consentaient, précisément, à déchiffrer (voire à constituer) le particulier langage divin de l'âme. On se rappellera comment Ignace avait insisté sur le rôle neutre du directeur avec l'objectif "que le Créateur et Seigneur se communique à l'âme pieuse ${ }^{51}$ " (Loyola $1969, \mathbb{1 5}$ ), précisant que le directeur devait assumer l'attitude indiquée à laisser « opérer inmediate le Créateur avec la créature, et la créature avec le Créateur ». En fait, c'est dans ce but qu'il devait s'abstenir de donner une quelconque suggestion à l'exercitant qui pouvait l'induire, par exemple, «à

49. Voir surtout Fernandez Martìn (1983) et Ortega-Costa (1980).

50. Voir Mongini (2011, 45-81).

51. De même pour les citations qui suivent. 
la pauvreté ou à la promesse » de "continence, virginité, état religieux et perfection évangélique». Et, toutefois, c'est précisément ce rapport direct et immédiat de l'exercitant avec Dieu qui soulevait des suspicions tenaces pour ses affinités évidentes avec la pratique du dexamiento ou dejamiento — l'abandon à Dieu dans le recueillement intérieur, prémisse pour accéder à son amour et à l'illumination — typique des alumbrados de Toledo ${ }^{52}$. De plus, la prohibition explicite, faite au directeur, d'orienter de quelques façons que ce soit l'exercitant vers des choix en soi tout à fait légitimes et — d'une manière ou d'une autre - en lien avec les coutumes ecclésiastiques, soulevait des suspicions quant à une certaine dévaluation implicite de certaines pratiques pourtant acceptées et reconnues par l'Église. C'est précisément sur cette quinzième Annotation que s'était arrêté le dominicain fray Tomás Pedroche dans sa Censura aux Exercices (1553), qui affirmait:

A mi ver, clara y abiertamente esta doctrina es de dejados y alumbrados, pues que pospuesto y dexado lo escrito, y pospuesta y dexada toda la enseñanza y doctrina que por buenas voces dan los buenos y sabios, se remiten $[\ldots]$ y dexan á lo que el spiritu y Dios dixeren allá en lo secreto del alma, [pour conclure que] si esta doctrina no es de dexados y alumbrados, querría ver una que fuesse dellos, para cotejarla con esta y ver cuanta distancia ay de la una a la otra ${ }^{53}$.

Il importe de relever que cette dernière observation du dominicain, voilée d'un certain sarcasme, réassumait, au fond, l'énigme historique des Exercices, dont la proximité de méthodes et de contenus aux doctrines des alumbrados ne pouvait que faire surgir le doute obsédant qu'il n'y avait, au fond, entre les uns et les autres, aucune "distance ". Le très grand danger que l'alumbradismo revêtait aux yeux des plus intransigeants théologiens espagnols - pour sa capacité de miner de l'intérieur les conceptions, doctrines et hiérarchies sociales, comportements, liturgies et rituels consolidés — avec précisément en arrière-plan la crise religieuse du XvI ${ }^{\mathrm{e}}$ siècle et

52. Sur l'alumbradismo, voir à tout le moins Firpo (1990), Firpo (1994) et Pastore (2004).

53. «À mon avis, clairement et ouvertement, cette doctrine est de dejados et alumbrados, puisque, renvoyé et abandonné l'écrit, et renvoyé et abandonné tous les enseignements et doctrines qui au moyen de bons conseils donnent les bons et les sages, s'en remettent et abandonnent à ce que l'esprit et Dieu disent dans le secret de l'âme »; "si cette doctrine n'est pas dexados et alumbrados, je voudrais en voir une qui fusse leur, pour la comparer et voir combien de distance il y a entre une et l'autre » (Pedroche 1900, 510). 
la rencontre avec l'hétérodoxie luthérienne, en vint à générer des peurs obsédantes - «la orden de los alumbrados [es] màs universal ${ }^{54}$ » - et déclencha, conséquemment, une redéfinition radicalement étroite et rigide d'orthodoxie qui impliquait, dans le tourbillon de l'hérésie, chaque aspect connecté à l'intériorisation de l'expérience religieuse. À nouveau, il convient de faire appel au regard perçant de Melchor Cano (précurseur, et de fait, inspirateur de Pedroche) pour comprendre comment ce dialogue direct entre l'exercitant et son "Créateur et Seigneur » qui se situe au cœur des Exercices, était incompatible avec la nouvelle et agressive orthodoxie qui frappait toute forme de consultation intérieure avec Dieu parce qu'elle:

tiene sabor de la heregia de los alumbrados, los quales en todas las cosas humanas e divinas, consultando por si mesmos a Dios, por el don de consejo que todos los justos tienen, pensavan seer alumbrados de el espiritu sancto en todas sus consultas; e assí en effecto e platica excluyan el consejo de los hombres doctos, o a lo menos teníanlo por impertinente; lo mesmo quasi en substancia han tenido algunos lutheranos ${ }^{55}$.

De la sorte, le présupposé de fond était que ce dialogue intérieur avec la divinité était considéré comme hérétique, à savoir que "para acertar no ay otro camino que certo sea sino consultar a Dios que alumbre nuestra razón ${ }^{56}$ : le présupposé, justement, de tous les Exercices de saint Ignace et de la certitude de l'élection.

De la même façon, cette interdiction explicite de fournir des conseils à l'exercitant, précédée par l'indication au directeur d'exposer les points et l'histoire de chacun des exercices de manière succincte sur la base du principe général selon lequel «ce n'est de savoir beaucoup que l'âme est rassasiée et assouvie, mais de sentir et de goûter les choses intérieurement " (Loyola 1969, \$2). Bien que prudemment formulée, elle laissait émerger

54. «L'ordre des alumbrados est vraiment universel/partout»: Melchor Cano, Censura y parecer que dió el P. Mtro Fr. Melchor Cano contra el Instituto de los PP. Jesuitas, ms. British Library, Egerton 453, f. 91-105; une édition de cet important écrit de Cano, longtemps considéré comme perdu, a été donnée par l'ex-jésuite Miguel Mir, dans la polémique (Mir 1913, 626).

55. «Il a la saveur de l'hérésie des alumbrados, lesquels dans toutes les choses humaines divines, par eux-mêmes consultant Dieu, pour le don du conseil que tous les justes possèdent, pensent d'être illuminés par l'Esprit Saint dans chacune de leur consultation; et ainsi, dans les faits et dans les conversations, ils excluent les conseils des hommes doctes, ou pour le moins, le considèrent futile; pratiquement le même, en substance, ont retenu plusieurs luthériens» (Caballero 1871, 557).

56. «Pour atteindre la certitude, il n'y a pas d'autre chemin qui ne soit certain si ce n'est pas de consulter Dieu, qui illumine notre intelligence» (Caballero 1871, 557). 
une dévaluation implicite du savoir livresque, de la science des doctes et des letrados, et évoquait une attitude typique de l'alumbradismo: la polémique antiscolastique, le refus de l'enseignement des théologiens de profession et de tous ceux qui croient par relation et non par expérience. Cela était aussi une conséquence de ce "consultar a Dios" personnel et subjectif que, comme avait bien vu Melchor Cano, "escluye el magisterio de los hombres doctos e prudentes » et la médiation de l'Église. «Sentir et goûter intérieurement ", avait écrit Loyola; et c'était précisément cela la doctrine des alumbrados, des «barones espirituales », à la différence des «theologos escolasticos ", "por experiencia e gusto saben las cosas divinas e humanas ${ }^{57}$ ». Donc, à bien y voir, sous la patine anonyme et ambivalente du langage et des formules des Exercices, apparaissent plusieurs points de contact avec les noyaux caractéristiques de l'alumbradismo. Et l'élection ignacienne, un des buts essentiels vers lequel était orientée l'expérience religieuse des Exercices, non seulement représentait un moment dans lequel le dialogue direct de l'exercitant avec Dieu était dirigé explicitement pour en consulter la volonté, mais apparaissait l'emblème même de cette aspiration typiquement alumbrada à instaurer dans l'homme la "gobernación del espíritu santo ${ }^{58}$ », unique guide, authentique et certain, de la conscience.

Enfin, en plus des éléments de contenu relevés jusqu'ici et pour compléter ces brèves considérations en regard de l'historicisation de la pensée religieuse de Loyola, il est nécessaire d'aborder un autre élément, duquel, au cours de ces pages, on a tenté de fournir quelques exemples. Un facteur central et substantiellement non exploré par la recherche est le fait que toutes sources jésuites antiques, y compris celle de Loyola, présentent un langage aux caractéristiques spécifiques qu'il faut décrypter patiemment, et dont il faut d'abord identifier les causes historiques. En effet, il est possible de formuler l'hypothèse selon laquelle les polémiques récurrentes, les accrochages et les accusations provenant de toute part, les procès et les enquêtes sur lui et sur la Compagnie, aient contribué de façon décisive à faire en sorte que Loyola élabore un langage particulier, fortement ordinaire et anodin. Un langage "commun ", inséré dans un "modo de hablar» propre aux jésuites, par lequel dissimuler les contenus les plus controversés

57. «Par expérience et goût, ils connaissent les choses divines et humaines » (Caballero 1871, 577).

58. La «gouvernance de l'esprit saint »: de Valdés $(1885,172)$; voir Firpo (1990) et de Valdés (1994). 
et radicaux de sa proposition religieuse. Un langage revêtant ainsi l'expérience jésuite, exprimée en premier lieu dans les Exercices, mais aussi dans les Constitutions et dans la généralité des sources antiques, de formes pieuses et prudentes, saupoudrées de formules de respect et d'obédience face à la hiérarchie de l'Église et empreintes de respect et d'éloge quant aux pratiques, dévotions, liturgies communément acceptées des ordres religieux monastiques et mendiants (desquels, par ailleurs, les pratiques et dévotions étaient traditionnellement le monopole $)^{59}$. Loyola mettait à disposition toutes les ressources de la perfection intérieure, de la méditation, de la prière mentale, ainsi que la doctrine et les instruments du discernement spirituel. Toutefois, il ne renonçait pas à proposer un modèle (la Compagnie de Jésus) — qui entendait se différencier du modèle institutionnel des ordres monastiques et conventuels, en laissant de côté plusieurs de leurs pratiques - et une typologie d'expérience religieuse (précisément celle des Exercices) - destinée à tous sans distinction, en particulier aux laïcs: il détruisait ainsi les anciennes coutumes culturelles et sociales toujours renouvelées qui avaient érigé une barrière entre les spécialistes (ou les professionnels) de la prière et la gente commune (ces «populares» qui, selon Melchor Cano, devaient se limiter à la «vie active»).

Ainsi, le problème historique des Exercices était représenté par le fait qu'ils constituaient un moyen d'accès à un ample éventail d'expériences religieuses orientées, sans équivoque, au contact direct avec la divinité, à la consultation sans médiation de la volonté divine et à l'illumination intérieure comme dernière certification de l'inspiration et des choix de l'individu avec, en arrière-fond, un contexte historique marqué de manière croissante par la rencontre entre des regroupements confessionnels opposés. En effet, le conflit entre orthodoxie et hérésie poussait à ériger et à radicaliser les positions et les orientations et contribuait non seulement à multiplier les hérésies, mais également à la construction en parallèle d'une nouvelle orthodoxie. Orthodoxie beaucoup plus intransigeante à l'égard des idées, concepts, mouvements et expériences religieuses, dont les conte-

59. Les célèbres et controversées «Règles [...] pour sentir vraiment avec l'orthodoxe Église» ou Règles «pour la vrai direction que nous devons suivre dans l'Église militante ", reflètent d'une façon exemplaire ces prescriptions et codes du langage jésuite (voir Loyola 1969, $\mathbb{3} 352-370$ ), et avec lesquelles se concluaient les Exercices. Sans pouvoir s'arrêter dans ces pages sur celles-ci, il est nécessaire d'au moins souligner qu'elles constituent, à bien y voir, un document extraordinaire. De celui-ci tirent leurs origines les particularités et les caractéristiques de la Compagnie de Jésus, ainsi que ses attitudes de fond, bien qu'exprimées dans une forme indirecte et dissimulée. 
nus ou dont le langage pouvait faire allusion ou évoquer non seulement des doctrines, mais aussi des formules et des termes utilisés par les hérétiques modernes, jusqu'à couvrir de l'ombre de la suspicion et de l'hétérodoxie le langage évangélique tout comme les paroles de saint Paul, devenues désormais imprononçables ${ }^{60}$. De ce problème historique fondamental dérivaient, à bien y regarder, les caractéristiques principales des Exercices ignaciens - et de la proposition religieuse jésuite - qui devaient, en même temps, garantir son adhésion à une orthodoxie toujours plus rigide et rigoureuse, et conserver et transmettre les noyaux essentiels et originaires d'une expérience religieuse, formellement marqués par des traits spiritualistes et illuministes et pourtant largement irréductibles à la nouvelle orthodoxie, de matrice contre-réformiste, qui se renforçait durement à travers mille conflits et disputes.

Il n'est donc pas surprenant, sur la base de ces brèves considérations, de constater les développements suivants (au prix de reléguer toujours plus au second plan les aspects proprement spiritualistes de la démarche d'Ignace): 1) avoir omis ou nuancé les noyaux théologiques les plus controversés, les enveloppant dans une réserve toujours plus dense ${ }^{61}$; 2) avoir reconstruit et redéfini, sur la base d'exigences nouvelles et de pressions croissantes, l'autoconscience historique et la mémoire de l'ordre (Mongini 2011, 22-44), ainsi que ses particularités institutionnelles et de gouvernance interne (Catto 2010); 3) avoir mis l'accent sur les aspects pénitentiels, la conversion au péché, la confession et la prédication - c'està-dire sur la dimension horizontale et communicative, simplement pastorale du modèle jésuite; 4) avoir parallèlement limité, à tout le moins sur le plan normatif, l'accès aux Exercices à certaines catégories de personnes; 5) avoir insisté sur les devoirs d'obéissance envers les hiérarchies, élaborant en même temps un langage toujours plus «commun» sous lequel évasivement envelopper et transmettre quand même quelque chose du patrimoine religieux originaire.

Finalement, c'est grâce à ces ajustements, en amenant les institutions ecclésiastiques et les adversaires à croire que «l'oraison de Loyola ne veut

60. Voir les surprenantes affirmations de Melchor Cano, dans Caballero (1871, 585).

61. Il est possible d'en trouver quelques traces dans les vives discussions et échanges toutes internes à la Compagnie, jusqu'à l'intervention, à sa façon résolutive, du Saint Office - qui accompagnent la rédaction de la partie proprement théologique et doctrinale de la Ratio studiorum, en particulier le célèbre De delectu opinionum: un problème qui traîne depuis des décennies, que l'on ne peut que mentionner; voir Bianchi (2002, 32-36) pour quelques réflexions d'introduction. 
pas générer une théophanie en soi ${ }^{62}$ " (Motta 2005, 62) que les jésuites et les Exercices spirituels purent être reconduits dans les confins bien gardés de l'orthodoxie de la Contre-Réforme. Placés à présent sous le signe d'une pieuse et rassurante dévotion, ils purent donc se frayer leur chemin pluriséculaire dans la spiritualité catholique par le biais des missions populaires, des congrégations mariales et du solide réseau de collèges jésuites (avec l'innovante Ratio studiorum) ${ }^{63}$.

\section{Références}

Concordancia ignaciana (1996), Bilbao.

Barthes, R. (1977) [français 1971], Sade, Fourier, Loyola. La scrittura come eccesso / trad. par L. Lonzi, Turin/Paris, Seuil.

Bianchi, A. (2002), dir., Ratio atque institutio studiorum Societatis Iesu, Milan, Biblioteca Universale Rizzoli.

BRIZZI, G. P. (1981), La "Ratio studiorum». Modelli culturali e pratiche educative dei gesuiti in Italia tra Cinque e Seicento, Rome, Bulzoni.

Caballero, F. (1871), Conquenses illustres, vol. II, Melchor Cano, Madrid.

Cano, M., Censura y parecer que dió el P. Mtro Fr. Melchor Cano contra el Instituto de los PP. Jesuitas, ms. British Library, Egerton 453, f. 91-105.

Catтo, M. (2010), "Le Costituzioni gesuitiche tra tradizione religiosa e modernità ", dans T. McCoog, dir., Ite inflammate omnia. Selected historical papers from conferences held at Loyola and Roma, Rome, p. 159-182.

Fabre, P.-A. (1992), Le lieu de l'image. Le problème de la composition de lieu dans les pratiques spirituelles et artistiques jésuites de la seconde moitié du XVI siècle, Paris, Vrin.

Fernandez Martìn, L. (1983), "Iñigo de Loyola y los alumbrados ", Hispania sacra, 35, p. 585-680.

Firpo, M. (1990), Tra alumbrados e «spirituali». Studi su Juan de Valdés e il valdesianesimo nella crisi religiosa del '500 italiano, Firenze, Olshki.

62. Aux pages 58-65, se trouve un examen convaincant des modalités par lesquelles les Exercices devinrent « un pivot robuste de la catholicisation de masse » (Motta 2005, 60). Sur l'absence de «théophanie » dans les Exercices, voir également Barthes (1977, 34).

63. Voir, par exemple, Brizzi (1981); Hinz et al. (2004). 
(1993), Riforma protestante ed eresie nell'Italia del Cinquecento, Roma-Bari, Laterza.

(1994), «Introduzione ", dans J. DE VALDÉs, Alfabeto cristiano, Turin, Einaudi, p. VII-CL.

(2008), "Juan de Valdés. "Dottore e pastore di persone nobili e illustri” ", dans G. Zarri, dir., Storia della direzione spirituale, vol. III, L'età moderna, Brescia, Morcelliana, p. 183-220.

GIL, D. (1971), La consolación sin causa precedente, Rome, CIS.

Gouvernaire, J. (1980), Quand Dieu entre à l'improviste. L'énigme de la consolation sans cause, Paris, Desclée de Brouwer.

Hinz, R., Righi, R. et Zardin, D. (2004), dir., I gesuiti e la Ratio studiorum, Rome, L. Ceriotti.

Jurado, M.R. (1997), Il discernimento spirituale. Teologia, storia, pratica, Cinisello Balsamo, Milan.

LAInEz, J. (1943), Epistola de Patre Ignatio, dans Fontes narrativi, I, MHSI: Rome.

Loyola, S. Ignatil (1943), Acta Patris Ignatii, dans Fontes narrativi, I, MHSI : Rome.

(1943a), Fontes narrativi, I, MHSI : Rome.

(1969), Exercitia spiritualia, MHSI : Rome.

(2000), Esercizi spirituali. Testi complementari, éd. par H. Alfonso S.I., Rome.

(2007), Journal des motions intérieures, éd. par P.-A. FABRE, dir., Paris, Lessius.

Mir, M. (1913), Historia interna documentada de la Compania de Jesús, publié par Jaime Ratés Martín, Madrid, vol. II, pp. 621-631.

Mondez, J. (1977), «Consolation sans cause et effusion de l'Esprit », dans Christus, 96, p. 482-491.

Mongini, G. (2011), Ad Christi similitudinem. Ignazio di Loyola e i primi gesuiti tra eresia e ortodossia, Alessandria, Edizioni dell'Orso.

(2012), Poteri carismatici e dottrine di perfezione. Brigida Morello di Gesù (1610-1679): un'esperienza di santità nel Seicento italiano, Alessandria, Edizioni dell'Orso.

MotтA, F. (2005), Bellarmino. Una teologia politica della Controriforma, Brescia, Morcelliana. 
Ortega-Costa, M. (1980), "San Ignacio en el "Libro de alumbrados": nuevos datos sobre su primer proceso ", dans Arbor, 107, p. 163-174.

PAstore, S. (2004), Un'eresia spagnola. Spiritualità conversa, alumbradismo e Inquisizione (1449-1559), Firenze, Olshki.

Pedroche, T. (1900), "Censura [...] contra los exercicios del P. M. Ignacio", dans A. Polanco, Vita Ignatii Loyolae (=Chronicon), vol. III, Madrid.

Plazaola, J. (1998), dir., Las fuentes de los Ejercicios espirituales de San Ignacio, Bilbao, Mensajero.

Ribadeneira, P. De (1589), Tratado de la tribulación, Madrid.

VAldÉs, J. DE (1885), Comentario a los Salmos, Madrid. (1994), Alfabeto cristiano, Turin.

VRIEs, P. P. DE (1979), Discernement des esprits. Ignace de Loyola, Paris.

\section{Résumé}

L'article traite de la question du discernement intérieur dans la pensée du fondateur de la Compagnie de Jésus, Ignace de Loyola. Malgré l'existence d'une vaste littérature sur le discernement ignacien, certains problèmes demeurent, notamment celui de la relation entre la théorie du discernement, exposée dans les Exercices spirituels d'Ignace, et la pratique. L'article a pour but de retracer le système des «règles du discernement» dans les Exercices, en clarifiant certains malentendus herméneutiques concernant les concepts (surtout en référence à la lecture faite par Roland Barthes) et l'histoire du $\mathrm{XVI}^{\mathrm{e}}$ siècle (où l'expérience religieuse d'Ignace de Loyola trouve ses racines). Aux alentours de 1550, la méthode du discernement proposée dans les Exercices a suscité de nombreuses polémiques, notamment en Espagne. Ici, à la question de l' «élection ", qui requiert la mise en pratique du discernement, s'est ajoutée celle de la relation entre spiritualisme, hérésie et orthodoxie, jusqu'à la remise en question des fondements de l'expérience religieuse jésuite dans son ensemble. 


\section{Abstract}

This paper deals with the topic of inner discernment in the thought of Ignatius of Loyola, founder of the Society of Jesus. In spite of the extended literature on the Ignatian discernment, several questions still need to be answered, especially the one pertaining to the relationship between discernment's theory (explained in the Ignatius'Spiritual Exercises) and praxis. This article aims at retracing the system of "discernment's rules" in the Exercices and at clarifying some hermeneutical misinterpretations with regards to concepts (particularly the Roland Barthes'reading) and to the $\mathrm{XVI}^{\text {th }}$ century historical context (in which the Ignatius'religious experience finds its roots). Around the year 1550, the method of discernment described in the Exercices sparked various polemics, particularly in Spain. Here, the issue of "election", that requires discernment's activation, merged with other issues, such as the relationship between spiritualism, heresy, and orthodoxy, to the extent that the very foundations of Jesuit religious experience were questioned. 